Gautam Dhar, MD'

Bijan Basak, MS (ENT)2

Ganesh Chandra Gayen, MS (ENT) ${ }^{2}$

Ritam Ray, MS (ENT)2

'Department of Community Medicine, Swasthya Bhawan, Salt Lake City, West Bengal, India

${ }^{2}$ Department of ENT, Burdwan Medical College,

West Bengal, India

\section{Auricular Perichondritis in a Tertiary Rural Hospital}

\begin{abstract}
Objective: Auricular perichondritis refers to inflammation involving the perichondrium of the external ear. It is a very serious disease which may lead to permanent deformity of the pinna. We describe the predisposing factors, pathogenic organisms, interventions and residual deformities in a prospective consecutive sample of patients.
\end{abstract}

\section{Methods:}

Design: Prospective Cohort Study

Setting: Tertiary Rural Government Teaching Hospital

Participants: All patients presenting with auricular perichondritis for a period of one year between March 2011 and February 2012 were consecutively enrolled and a clinical history and demographic details were obtained. Routine hematologic, blood biochemical examinations and culture / sensitivity of discharge from the pinna were conducted, and empiric intravenous ciprofloxacin was administered and continued if confirmed by culture and sensitivity. Those sensitive to co-amoxiclav, ceftazidime or amikacin were shifted to those medications. Medications were shifted to oral forms when available and indicated by resolution of acute inflammation, wound healing and no growth on cultures. Parenteral medications were maintained until the same parameters were achieved. Surgical incision and drainage was also performed when indicated, followed by a standardized wound care regimen. Follow up was for six months ending with assessment of pinna deformity.

Results: Of the total study population of $50,76 \%$ were male and $24 \%$ were female; 15 75 years of age (range 60 years) displaying male predominance and clustering in the fourth decade of life. The most common predisposing factors were trauma from motor vehicle accidents (30\%) followed by high ear piercing (22\%). Pseudomonas aeruginosa (48\%) followed by Staphylococcus aureus (20\%) were the most common organisms isolated. All were managed with intravenous antibiotics but $76 \%$ also required surgical intervention. Sixty-eight percent developed residual deformities of the pinna with $50 \%$ being total and $18 \%$ being partial.

Conclusion: Auricular perichondritis is a frightening disease which requires early management. As Pseudomonas aeruginosa is the most common organism, antipseudomonal antibiotics should be started as early as possible. Despite medical and surgical intervention, residual deformities may ensue.

\section{Keywords: auricular perichondritis, pinna, trauma, Pseudomonas aeruginosa}


Perichondritis refers to an inflammation of the perichondrium, a layer of connective tissue which surrounds cartilage. Auricular perichondritis, the commonest form, involves the pinna due to infection of a traumatic or surgical wound or the deep spread of superficial inflammation. This type of disease often leads to residual deformity, and its incidence in our experience seems to be increasing. ${ }^{1}$ The present study was conducted to determine the predisposing factors, pathogenic organisms, interventions and residual deformities of the disease in a local rural setting in West Bengal, India.

\section{MATERIALS AND METHODS}

With institutional ethical review board approval, this prospective series was conducted in the Department of ENT, Burdwan Medical College and Hospital, Burdwan. All patients presenting with auricular perichondritis over a period of one year from March 2011 to February 2012 were consecutively enrolled after obtaining informed consent. No other inclusion or exclusion criteria were applied.

A detailed clinical history including demographic details, medical history (diabetes mellitus, autoimmune diseases, recurrent otitis), recent medical history related to current illness (any surgical intervention on the infected ear within one month of the current hospitalization) possible predisposing events (trauma, acupuncture), clinical data (body temperature and physical signs such as local hyperemia, swelling, discharge and tenderness), were taken from each patient. They were all examined properly to exclude other pathologies.

All patients subsequently underwent routine hematologic and blood biochemical examinations and discharge from the pinna was sent for culture and sensitivity. All patients were treated with empirical intravenous ciprofloxacin after admission, and continued on ciprofloxacin if clinical response and culture and sensitivity were confirmed. Those sensitive to co-amoxiclav, ceftazidime or amikacin were shifted to these medications. Step-down to oral medications (ciprofloxacin or co-amoxiclav) was done when signs of acute inflammation resolved, the wound appeared healthy and culture revealed no growth. Those on ceftazidime or amikacin were maintained on parenteral medications until proper wound healing had been achieved.

When associated with hyperemia, fluctuant swelling of the pinna and aspirated pus, surgical incision and drainage was also performed. Regular wound dressing was done in every patient with hydrogen peroxide (20\%) sprayed into the wound, rinsed with normal saline, washed with povidone iodine $10 \%$ (betadine), rinsed with normal saline, swabbed with tetrachlorodecaoxide 1:55 dilution (oxoferin) with a cotton applicator and finally coated after 20 minutes with mupirocin $2 \%$ (T-bact) ointment. As the wound appearance improved, hydrogen peroxide, followed by povidone iodine and normal saline were successively discontinued. Tetrachlorodecaoxide was continued until crust formation, and mupirocin was used until epithelialisation.

All the patients were followed up for six months after control of infection for assessment of pinna deformity as a sequelae of perichondritis. Deformity of pinna following perichondritis was classified into three: no deformity, partial deformity (part of the pinna is deformed) and total deformity (whole pinna is deformed).

The data was tabulated and analyzed manually using means and percentages.

\section{RESULTS}

A total of 50 patients were studied over a period of one year. Of these, $38(76 \%)$ were male and $12(24 \%)$ were female. Their ages ranged from 15-75 years, with a range of 60 years. Most patients (38 patients, 76\%) were within the age group between 30-50 years.

Among the predisposing factors, 15 cases (30\%) were from trauma due to road traffic accidents, followed by ear piercing (11, $22 \%$ ). (Table 1) Pseudomonas aeruginosa was the most common pathogenic organism isolated from the diseased pinna (48\%) followed by Staphylococcus aureus (20\%). Polymicrobial infection was found in $8 \%$. (Table 2)

Table 1. Predisposing factors for perichondritis of the auricle

\begin{tabular}{|l|c|c|}
\hline $\begin{array}{l}\text { Predisposing } \\
\text { factors }\end{array}$ & $\begin{array}{l}\text { No of Cases } \\
(\mathbf{n}=\mathbf{5 0})\end{array}$ & Percentage (\%) \\
\hline Road traffic accident & 15 & 30 \\
\hline Ear piercing & 11 & 22 \\
\hline Burn & 8 & 16 \\
\hline Post - surgical & 7 & 14 \\
\hline Diabetes & 5 & 10 \\
\hline Allergic reaction & 4 & 8 \\
\hline Total & 50 & 100 \\
\hline
\end{tabular}


Table 2. Pathogenic organisms isolated from perichondritis

\begin{tabular}{|l|c|c|}
\hline \multicolumn{1}{|c|}{$\begin{array}{c}\text { Pathogenic } \\
\text { isolates }\end{array}$} & $\begin{array}{l}\text { No of Cases } \\
(\mathbf{n = 5 0 )}\end{array}$ & Percentage (\%) \\
\hline Pseudomonas aeruginosa & 24 & 48 \\
\hline Staphylococcus aureus & 10 & 20 \\
\hline Enterococcus faecalis & 5 & 10 \\
\hline Streptococcus group A & 3 & 6 \\
\hline Polymicrobial infection & 4 & 8 \\
\hline Klebsiella pneumonia & 2 & 4 \\
\hline Candida spp & 2 & 4 \\
\hline Total & 50 & 100 \\
\hline
\end{tabular}

Twelve patients (24\%) responded to antibiotic therapy alone while 38 patients (76\%) required additional surgical incision and drainage. Thirty patients (60\%) responded with empiric ciprofloxacin and their culture sensitivity reports confirmed sensitivity to ciprofloxacin in twenty nine (29) or 58\%. Eleven patients (22\%) whose reports revealed sensitivity to coamoxiclav were shifted to it but only 10 or $20 \%$ were continued on a full course. One patient each on initial ciprofloxacin and co-amoxiclav subsequently had Candida spp on culture, prompting us to cease the respective antibiotics and continue with topical wound care alone. Six patients required amikacin and three (3) were shifted to ceftazidime. (Table 3) All patients on ciprofloxacin and co-amoxiclav were eventually shifted to oral forms, and those on amikacin and ceftazidime were maintained on parenteral forms. All wounds healed sufficiently to cease oral or intravenous antibiotics within 20 to 46 days.

After six months follow-up, 34 patients (68\%) developed residual deformity. These were further subdivided into 25 (50\%) with total deformity of the pinna and 9 (18\%) with partial deformity. (Table 4)

Table 4. Deformity following perichondritis

\begin{tabular}{|l|c|c|}
\hline Type of deformity & $\begin{array}{l}\text { No of Cases } \\
(\mathbf{n = 5 0 )}\end{array}$ & Percentage (\%) \\
\hline No Deformity & 16 & 32 \\
\hline Partial deformity & 25 & 50 \\
\hline Total deformity & 9 & 18 \\
\hline Total & 50 & 100 \\
\hline
\end{tabular}

Table 3. Pathogenic Organisms and Antibiotic Sensitivities

\begin{tabular}{|c|c|c|c|c|c|c|c|c|c|c|c|c|}
\hline \multirow{3}{*}{$\begin{array}{l}\text { Pathogenic } \\
\text { Organisms }\end{array}$} & \multicolumn{12}{|c|}{ Antibiotic Sensitivity** } \\
\hline & \multicolumn{3}{|c|}{ Ciprofloxacin } & \multicolumn{3}{|c|}{ Co-amoxiclav } & \multicolumn{3}{|c|}{ Amikacin } & \multicolumn{3}{|c|}{ Ceftazidime } \\
\hline & $\mathbf{S}$ & I & $\mathbf{R}$ & $\mathbf{S}$ & I & $\mathbf{R}$ & $\mathbf{S}$ & I & $\mathbf{R}$ & $\mathbf{S}$ & I & $\mathbf{R}$ \\
\hline $\begin{array}{l}\text { Pseudomonas } \\
\text { aeuroginosa }\end{array}$ & 22 & & 2 & 0 & & 24 & 1 & & 23 & 1 & & 23 \\
\hline Staph aureus & 3 & & 7 & 7 & & 3 & 0 & & 10 & 0 & & 10 \\
\hline Enterococcus faecalis & 3 & & 2 & & & 5 & 2 & & 3 & & & 5 \\
\hline Streptococcus Group A & 0 & & 3 & 0 & & 3 & 3 & & 0 & 0 & & 3 \\
\hline Klebsiella pneumoniae & 0 & & 2 & 0 & & 2 & 0 & & 2 & 2 & & 0 \\
\hline Polymicrobial Infection* & 1 & & 3 & 3 & & 1 & 0 & & 4 & 0 & & 4 \\
\hline Total & 29 & & 19 & 10 & & 38 & 6 & & 42 & 3 & & 45 \\
\hline
\end{tabular}

*Organisms in polymicrobial infection

Patient 1- E.coli, Enterobacter, Shigella, Proteus:

Patient 2- H.influenzae, Shigella, Enterobacter:

Patient 3- Proteus, Shigella, Enterobacter:

Patient 4- Salmonella, H.influenzae, E.coli:
Sensitive to ciprofloxacin.

Sensitive to co-amoxiclav.

Sensitive to co-amoxiclav

Sensitive to co-amoxiclav

${ }^{* *}$ One patient each had Ciprofloxacin and Co-amoxiclav discontinued after Candida spp was grown; totalling 50 patients (48 reflected in this table). 


\section{DISCUSSION}

Perichondritis of the auricle is a frightening and perturbing complication of the traumatized ear that can lead to residual deformity. ${ }^{1}$ It usually results from trauma which may include injuries following road traffic accidents, post-surgery. ${ }^{2,3}$ and burns. ${ }^{2,3,4,5}$ Contaminated wounds following road traffic accidents are more prone to perichondritis of the pinna. The popularity of high ear piercings has been increasing among teenagers ${ }^{6}$ and when performed by untrained, unqualified persons without maintaining aseptic technique, may increase the incidence of aural perichondritis, as seen in our study.

Skin moisture fosters the proliferation of the most common causal agent.' Patients usually present with unbearable pain, erythema of pinna and rise of body temperature. If left untreated, the disease progresses as diffuse edema of the pinna and subsequent abscess formation leading to cartilage necrosis and cauliflower deformity.

Dowling et al., ${ }^{8}$ Apfelberg et al. ${ }^{9}$ and Bassiouny ${ }^{3}$ found that Pseudomonas aeruginosa is the most common pathogenic organism responsible for this disease followed by Staphylococcus aureus ${ }^{10}$ which was consistent with our findings. These pathogens are usually sensitive to ciprofloxacin or co-amoxiclav, as seen in our study. Other pathogens include Enterococcus faecalis, Streptococcus group A and Klebsiella pneumonia which may be treated with ceftazidime and amikacin, again reflected in our study.

Four patients had polymicrobial infection with Escherichia coli, Enterobacter, Shigella, Proteus, H.influenzae and Salmonella found on cluture. Of the four patients, three were sensitive to co-amoxiclav and one was sensitive to ciprofloxacin.

Auricular perichondritis not only involves the perichondrium but also the chondral cartilage. The regeneration of damaged cartilage is difficult and necrotic chondrocytes will eventually be replaced by dense fibrosis and scar formation, which will cause consequent ear deformity. ${ }^{11}$ In our study, residual deformity of pinna was found in $68 \%$ of cases after six months follow-up.

Indeed, auricular perichondritis is a very frightening and frustrating disease. It requires prompt intervention and management, despite which permanent remodelling of the pinna may occur. Perhaps the best way to prevent sequelae of this disease is still highlighting education on the risk factors and early intervention with administration of proper medications. Future studies should investigate the role allergic reactions may have as important contributing factors to auricular perichondritis, as we were unable to do so in this study.
REFERENCES

1. Davidi E, Paz A, Duchman H, Luntz M, Potasman I. Perichondritis of the auricle: analysis of 114 cases. Isr Med Assoc J 2011 Jan;13(1): 21-24.

2. Prasad HK, Sreedharan S, Prasad HS, Meyyappan MH, Harsha KS. Perichondritis of the auricle and its management. J Laryngol Otol 2007;121(6):530-4.

3. Bassiouny A. Perichondritis of the auricle. Laryngoscope 1981;91(3):422-31

4. Prasad KC, Karthik S, Prasad SC. A comprehensive study on lesions of the pinna, Am J Otolaryngol 2005; 26(1): 1-6

5. Templer J, Renner GJ. Injuries of the external ear. Otolaryngol Clin North Am 1990 23(5): 1003-18.

6. More DR, Seidel JS, Bryan PA. Ear piercing techniques as a cause of auricular chondritis. Pediatr Emerg Care. 1999;15(3):189-92.

7. Das P. Piercing the cartilage and not the lobules leads to ear infections. Lancet Infect Dis 2002;2(12):715

8. Dowling JA, Foley FD, Moncrief JA. Chondritis in the burned ear. Plast Reconstr Surg $1968 ; 42(2): 115-22$.

9. Apfelberg DB, Waisbren BA, Masters FW, Robinson DW. Treatment of chondritis in the burned ear by the local instillation of antibiotics. Plast Reconstr Surg 1974;53(2): 179-83.

10.Stirn A. Body piercing: medical consequences and psychological motivations. Lancet 2003; 361(9364):1205-15.

11.Stroud MH. A simple treatment for suppurative perichondritis. Laryngoscope 1963 73(5):556-63 